\title{
Unsaturated Polyester Resin as Non-formaldehyde Adhesive Used in Bamboo Particle Boards
}

Zhenzeng Wu, ${ }^{1}$ * John Tosin Aladejana, ${ }^{2}$ Shuqiong Liu, ${ }^{1}$ Xinhuai Gong, ${ }^{1}$ Xiaodong(Alice) Wang, ${ }^{3}$ and Yongqun $\mathrm{Xie}^{2}$, *

${ }^{1}$ The College of Ecology and Resource Engineering, Wuyi University, No. 16, Wuyi Avenue, Wuyishan City, Fujian 354300, P.R. China

2 The College of Material Engineering, Fujian Agriculture and Forestry University, 15 Shangxiadian Road, Fuzhou, Fujian 350002, P.R. China

${ }^{3}$ The Department of Wood and Forest Sciences, Laval University, Quebec G1V 0A6, Canada

\section{E-mail for authors:}

John Tosin Aladejana - E-mail: jtaladejana@futa.edu.ng;

Shuqiong Liu - E-mail: liushuqiong123456@163.com;

Xinhuai Gong - E-mail: wyu_gxh@163.com;

Xiaodong(Alice) Wang - E-mail: xiaodong.wang@sbf.ulaval.ca

\section{* Corresponding Author:}

Zhenzeng Wu - ORCID: https://orcid.org/0000-0002-9046-9667; E-mail: zhenzeng.wu@wuyiu.edu.cn Yongqun Xie, Tel: +86 591 83789307. E-mail: fjxieyq@hotmail.com. 
Different adhesives such as UF+MDF ${ }^{1}$, citric $\mathrm{acid} / \mathrm{starch}^{2}$, silicon magnesium ${ }^{3}$, magnesium oxychloride 4 and soy-based adhesive ${ }^{5}$ on the property of IB used in bamboo particle boards were compared in Figure S1.

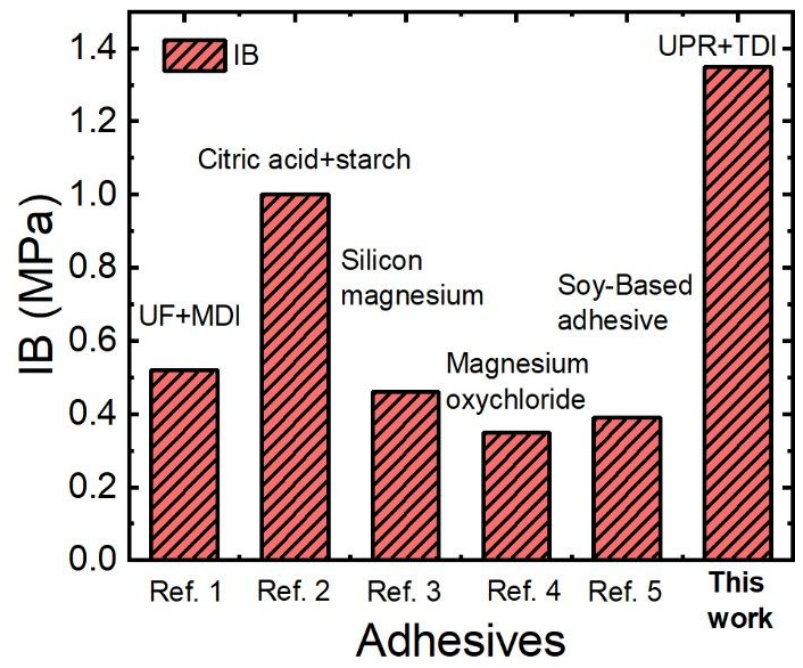

Figure S1. Comparison of IB with different adhesives.

\section{REFERENCE}

1. Ye, S., Study on Production Process of Bamboo Particleboard with Fine Bamboo Shaving Surface. China Wood-Based Panels 2021, 28 (2), 29-32.

2. Widyorini, R.; Umemura, K.; Kusumaningtyas, A. R.; Prayitno, T. A., Effect of Starch Addition on Properties of Citric Acid-bonded Particleboard Made from Bamboo. Bioresources 2017, 12 (4), 8086-8077.

3. Huang, Q.; Zhao, X.; Zheng, X.; Li, X., Preparation and properties of silicon magnesium cement with bamboo particle board. New Build. Mater. 2018, 45 (9), $25-$ 29.

4. Wang, Z.; Ma, L.; Fang, C.; Zhuang, Q.; He, L.; Yang, L., Experiment on Hot Pressing Technology for Magnesium Oxychloride Cement Banded Bamboo Particle Board. J. Zhejiang For. Sci. Technol. 2012, 32 (04), 48-50.

5. Peng, S.; Gui, C.; Lin, X.; Lan, C.; Zhang, Z., Study on Process Parameters of the Bamboo Particleboard Bonded by Soy-Based Adhesive. China For. Prod. Ind. 2017, 44 (09), 19-23. 